\title{
Temperature-Independent Fiber-Based Optical Phase Conjugation
}

\author{
J. Y. Huh, E. S. Son, S. B. Jun, and Y. C. Chung, Fellow, IEEE
}

\begin{abstract}
We have investigated the effect of temperature variation on the performance of fiber-based optical phase conjugation (OPC) module. The results show that the bandwidth of the fiber-based OPC module could be reduced significantly by temperature variation. However, it would be difficult to control the temperature of bulky fiber spool precisely. To solve this problem, we have proposed and demonstrated a simple method to compensate the bandwidth reduction (caused by temperature variation) by adjusting the pump power. Using this method, we could maintain the 1-dB bandwidth of fiber-based OPC module within $5 \%$ even when the ambient temperature was varied as much as $\pm 15{ }^{\circ} \mathrm{C}$.
\end{abstract}

Index Terms-Highly nonlinear fiber (HNLF), nonlinear phase shift, optical phase conjugation (OPC).

\section{INTRODUCTION}

$\mathbf{R}$ ECENTLY, there have been substantial efforts to increase the operating speed of wavelength-division-multiplexing (WDM) system to and beyond $40 \mathrm{~Gb} / \mathrm{s}$. However, in such a high-speed WDM system, the maximum transmission distance could be limited by the waveform distortions caused by chromatic dispersion and fiber nonlinearities. This problem could be solved by placing an optical phase conjugation (OPC) module in the middle of the transmission link [1]-[3]. However, for the use in WDM systems, it is essential for the OPC module to have a wide and uniform bandwidth. To achieve such a bandwidth, the fiber-based OPC module typically utilizes a pump laser operating at the zero-dispersion wavelength $\left(\lambda_{0}\right)$ of fiber [4], [5]. However, it has been well known that $\lambda_{0}$ is sensitive to temperature [6]. As a result, the bandwidth of fiber-based OPC module could be affected by the changes in ambient temperature. In this letter, we investigated the effects of temperature variation on the bandwidth of the fiber-based OPC module. We measured the variation of $\lambda_{0}$ in highly nonlinear fiber (HNLF) and dispersion-shifted fiber (DSF) while changing the fiber temperature, and evaluated the bandwidth of fiber-based OPC module as a function of temperature variation. The results show that the bandwidth of the fiber-based OPC module could be reduced significantly by temperature variations. For example, the 1-dB bandwidths of HNLF- and DSF-based OPC modules were reduced by $17 \%$ and $41 \%$, respectively, when the temperature was changed by merely $5{ }^{\circ} \mathrm{C}$. Thus, to suppress the bandwidth reduction within $5 \%$, the temperature should be controlled with accuracy better than $\pm 2{ }^{\circ} \mathrm{C}$ and $\pm 1{ }^{\circ} \mathrm{C}$ for the HNLF- and DSF-based OPC modules, respectively. However, it would be

Manuscript received January 10, 2006; revised May 15, 2006.

The authors are with the Department of Electrical Engineering, Korea Advanced Institute of Science and Technology, Daejeon 305-701, Korea (e-mail: ychung@ee.kaist.ac.kr).

Digital Object Identifier 10.1109/LPT.2006.879541

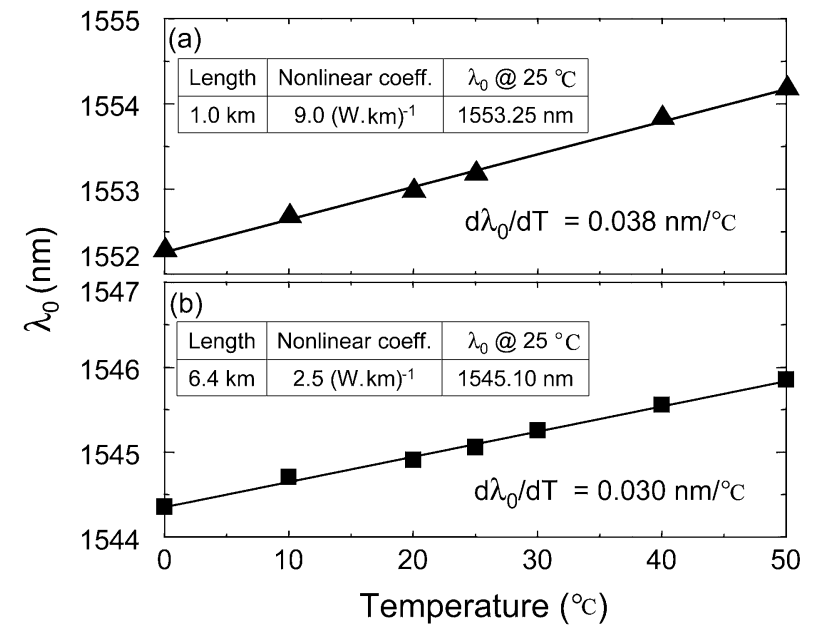

Fig. 1. Zero-dispersion wavelength $\left(\lambda_{0}\right)$ measured as a function of temperature. (a) HNLF. (b) DSF.

not only inefficient but also expensive to control the temperature of a bulky fiber spool within $1{ }^{\circ} \mathrm{C}-2{ }^{\circ} \mathrm{C}$. To solve this problem, we proposed and demonstrated a new technique for the temperature-independent fiber-based OPC module. This technique utilized the nonlinear phase shift obtained by adjusting the pump power to suppress the temperature dependency of fiber-based OPC. The results showed that this technique could maintain the $1-\mathrm{dB}$ bandwidth of HNLF-based OPC within $5 \%$ even when the ambient temperature was changed by $\pm 15^{\circ} \mathrm{C}$.

\section{EFFECT OF TEMPERATURE VARIATION ON FIBER-BASED OPC}

Fig. 1 shows the $\lambda_{0}$ 's of HNLF and DSF measured at various temperatures $\left(0{ }^{\circ} \mathrm{C}-50{ }^{\circ} \mathrm{C}\right)$ by using the four-wave-mixing technique [7]. The $\lambda_{0}$ 's of HNLF and DSF were 1553.25 and $1545.10 \mathrm{~nm}$ at $25^{\circ} \mathrm{C}$, and their temperature coefficients were 0.038 and $0.030 \mathrm{~nm} /{ }^{\circ} \mathrm{C}$, respectively. We implemented OPC modules by using 1-km-long HNLF and 6.4-km-long DSF. Fig. 2 shows the experimental setup used to measure the effect of temperature variation on the bandwidth of the fiber-based OPC module. The OPC medium (i.e., HNLF or DSF), placed in a temperature-cyclic chamber, was pumped by using a tunable laser. The pump wavelength was set to be identical to the $\lambda_{0}$ of HNLF or DSF measured at $25^{\circ} \mathrm{C}$. To suppress the stimulated Brillouin scattering (SBS), we modulated the pump laser at $2.5 \mathrm{~Gb} / \mathrm{s}$ by using an external phase modulator. The output power of pump laser was amplified to $\sim 19 \mathrm{dBm}$ by using an erbium-doped fiber amplifier (EDFA). We placed an optical bandpass filter (bandwidth: $1 \mathrm{~nm}$ ) at the output of EDFA to reduce the amplified spontaneous emission noise. A 


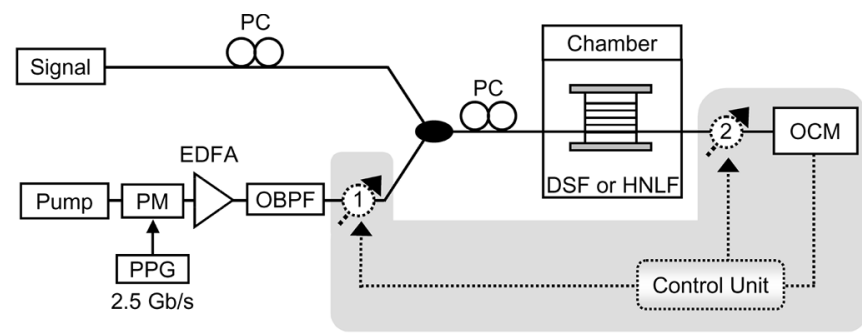

Fig. 2. Experimental setup to evaluate the effects of temperature variation on the efficiency and bandwidth of the fiber-based OPC module. The gray area represents the additional components used for the demonstration of the proposed temperature-independent fiber-based OPC module. (PM: phase modulator; PPG: pulse pattern generator; OBPF: optical bandpass filter.)

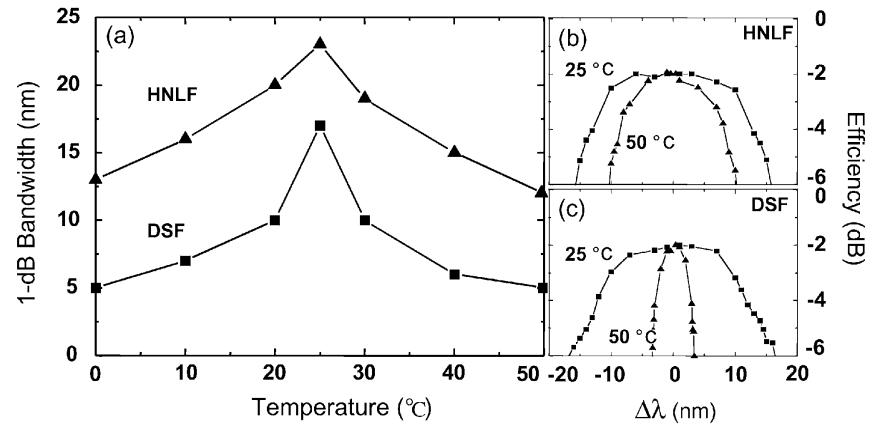

Fig. 3. (a) Measured bandwidths of HNLF- and DSF-based OPC modules as a function of temperature. The pump laser was set to operate at 1553.25 and $1545.10 \mathrm{~nm}$ for HNLF- and DSF-based OPC modules, respectively. (a) Measured efficiencies of DSF-based OPC modules at $25^{\circ} \mathrm{C}$ and $50^{\circ} \mathrm{C}$. (b) Measured efficiencies of HNLF-based OPC modules at $25^{\circ} \mathrm{C}$ and $50^{\circ} \mathrm{C}$.

polarization controller was used at the signal to maximize the efficiency of the OPC module. The pump was combined with the signal by using a $3-\mathrm{dB}$ coupler and sent to the OPC medium in the temperature-cyclic chamber. The output power of the signal incident on the OPC medium was $0 \mathrm{dBm}$. We measured the efficiency of the fiber-based OPC module while changing the signal's wavelength $\left(\lambda_{s}\right)$.

Fig. 3(a) shows the 1-dB bandwidth of OPC module measured while varying the temperature from $0{ }^{\circ} \mathrm{C}$ to $50^{\circ} \mathrm{C}$. In this experiment, we designed the OPC module to operate at $25{ }^{\circ} \mathrm{C}$ (i.e., the pump wavelength $\left(\lambda_{p}\right)$ was set to be identical to the $\lambda_{0}$ of HNLF (or DSF) measured at $25^{\circ} \mathrm{C}$ ). Thus, the bandwidth was decreased as we changed the temperature from $25^{\circ} \mathrm{C}$. For example, at $20^{\circ} \mathrm{C}$, the 1-dB bandwidth of HNLF-based OPC module was reduced from 23 to $19 \mathrm{~nm}$. This effect of temperature variation was more drastic for the DSF-based module (since the bandwidth was reduced from 17 to $10 \mathrm{~nm}$ ), although the temperature coefficient of HNLF was larger than that of DSF. This was mainly because the DSF-based OPC module required a much longer fiber than the HNLF-based module to obtain similar efficiency. Fig. 3(b) and (c) shows the bandwidths of DSFand HNLF-based OPC modules, respectively, measured at $25^{\circ} \mathrm{C}$ and $50^{\circ} \mathrm{C}$. The results clearly show that the bandwidths of OPC modules were substantially reduced at $50{ }^{\circ} \mathrm{C}$. At first sight, it appears that this problem could be solved easily by adjusting the pump wavelength to track the variation of $\lambda_{0}$. However, the pump wavelength should not be changed since it could also alter the wavelengths of the conjugated signals. Thus, to maintain the
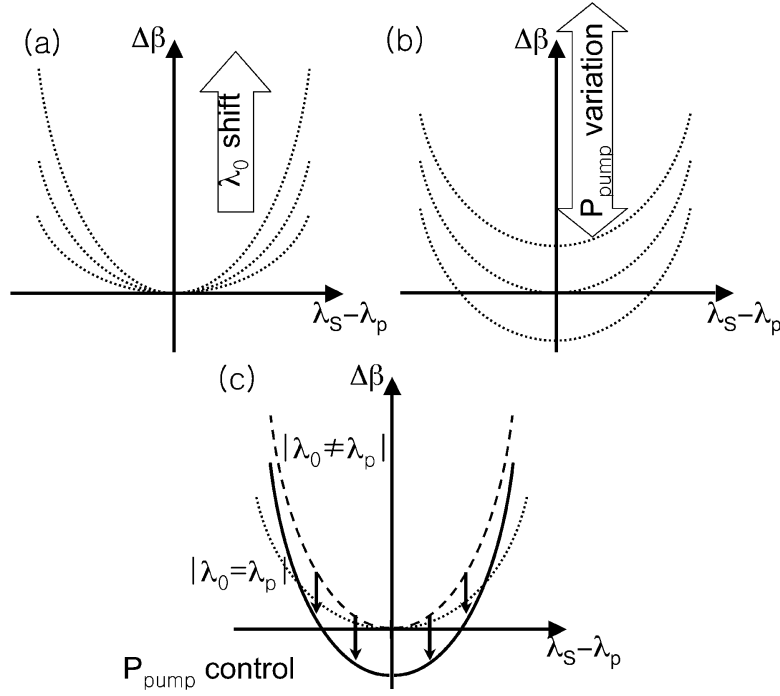

Fig. 4. Operating principle of the proposed temperature-independent fiberbased OPC module. (a) Changes of phase mismatch $(\Delta \beta)$ caused by $\lambda_{0}$ deviations (from $\lambda_{p}$ ), (b) changes of phase mismatch $(\Delta \beta)$ caused by pump power variations, and (c) compensation of phase mismatch $(\Delta \beta)$ caused by $\lambda_{0}$ deviations using pump power control.

bandwidth, it would be indispensable to control the temperature of OPC module. For example, to suppress the variation of 1-dB bandwidth within 5\%, the temperature of the HNLF-based OPC module should be regulated within $\pm 2{ }^{\circ} \mathrm{C}$. In case of using the DSF-based module, the temperature should be controlled within $\pm 1{ }^{\circ} \mathrm{C}$.

\section{TEMPERATURE-INDEPENDENT FiBER-BASED OPC}

To suppress the temperature dependency of fiber-based OPC module, it is necessary to control its temperature within $1{ }^{\circ} \mathrm{C}-2{ }^{\circ} \mathrm{C}$. However, it would be inefficient to control the temperature of a bulky fiber spool with such a precision. To solve this problem, we propose a new method based on the pump-power-induced nonlinear phase shift. Fig. 4 illustrates the operating principle of the proposed technique. Since the conjugated signals are generated by the four-wave mixing process between the pump and signals, the efficiency of fiber-based OPC module is dependent on their phase-matching condition. Thus, the maximum bandwidth can be achieved by operating the pump laser right at $\lambda_{0}$. However, if the temperature of OPC module is changed, $\lambda_{0}$ comes to deviate from the pump wavelength. This deviation would rapidly increase the amount of phase mismatch for the side channels, as shown in Fig. 4(a). On the other hand, the phase-matching condition can also be affected by the nonlinear phase shift caused by the high-power pump laser [8]. Depending on the pump power, this effect could either increase or decrease the amount of phase mismatch for the entire channels, as shown in Fig. 4(b). This is because the nonlinear phase shift is solely dependent on the pump power, and not on the wavelength. Thus, by adjusting the pump power, it should be possible to compensate the temperature-induced variation of the phase-matching condition. Fig. 4(c) shows that the amount of phase mismatch increases for the side channels as $\lambda_{0}$ deviates from the pump wavelength. However, as we increase the pump power, the entire phase mismatching 


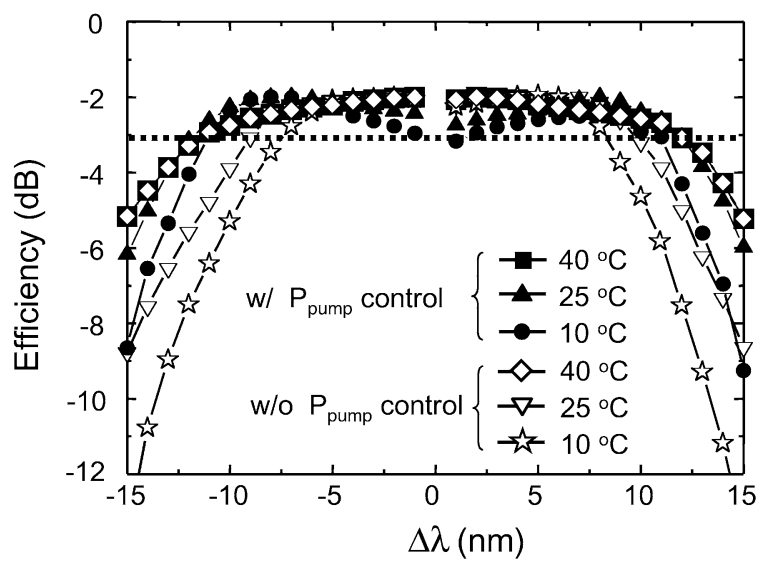

Fig. 5. Measured bandwidths of fiber-based OPC module (with and without using the pump power control) as a function of temperature variation.

curve becomes lowered. As a result, the bandwidth reduction caused by the temperature-induced variation of $\lambda_{0}$ can be compensated by adjusting the pump power (since it improves the phase-matching conditions for the side channels, while it degrades the conditions for the central channels).

We evaluated the performance of the proposed method by using the experimental setup in Fig. 2. For this evaluation, we have added an optical channel monitor (OCM), a couple of attenuators, and a control circuitry shown in the gray area to the conventional OPC module. We monitored the output powers of the conjugated signals by using OCM and adjusted the pump power to optimize the flatness of the OPC bandwidth by using Attenuator 1. Attenuator 2 was used to maintain the output power of the conjugated signal to be same regardless of the pump power. Fig. 5 shows the measured results while varying the temperature from $10{ }^{\circ} \mathrm{C}$ to $40{ }^{\circ} \mathrm{C}$. We first optimized the OPC module at $40{ }^{\circ} \mathrm{C}$ and then lowered the temperature. The results show that, when we did not use the proposed method, the efficiency of the side channel (at $\Delta \lambda=11.5 \mathrm{~nm}$ ) was reduced by $4 \mathrm{~dB}$. However, this efficiency was fully recovered when we increased the pump power from 19 to $23.7 \mathrm{dBm}$. The results also show that, using the proposed method, we could maintain the efficiency and the 1-dB bandwidth of this OPC module $(\sim 23 \mathrm{~nm})$ within $5 \%$ even when the temperature was varied as much as $\pm 15^{\circ} \mathrm{C}$. This result indicates the possibility of using the highest pump power at all time to prevent the problems associated with the bandwidth reduction caused by temperature variation. Thus, in this case, the bandwidth of the OPC module would be wider than needed most of the time. However, it should be noted that the unwanted four-wave mixing components could be amplified within this extra bandwidth, which, in turn, increase the full-width at half-maximum crosstalk and degrade the gain flatness due to pump depletion as the number of channels in the WDM system is increased [9]. Thus, to suppress the effects of temperature variation, it would be desirable to use the proposed technique than using the highest pump power at all time.

\section{SUMMARY}

We have investigated the effects of temperature variation on the bandwidth and efficiency of the fiber-based OPC module. The results show that the temperature of OPC module must be controlled precisely for the use in WDM systems. For example, to suppress the variation of $1-\mathrm{dB}$ bandwidth within $5 \%$, the temperature of the HNLF-based OPC module should be regulated within $\pm 2{ }^{\circ} \mathrm{C}$. For the same condition, the temperature of the DSF-based module should be controlled within $\pm 1{ }^{\circ} \mathrm{C}$. However, it would be not only inefficient but also expensive to control the temperature of a bulky fiber spool with such a precision. To solve this problem, we have developed a simple method to compensate the bandwidth reduction (caused by temperature variation) by adjusting the pump power. Using the proposed method, the 1-dB bandwidth of the HNLF-based OPC module could be maintained within $5 \%$ even when the ambient temperature was varied as much as $\pm 15^{\circ} \mathrm{C}$.

\section{REFERENCES}

[1] A. H. Gnauck, R. M. Jopson, and R. M. Deroiser, "10-Gb/s 360-km transmission over dispersive fiber using mid system spectral inversion," IEEE Photon. Technol. Lett., vol. 5, no. 6, pp. 663-666, Jun. 1993.

[2] S. Watanabe, T. Chikama, G. Ishikawa, T. Terahara, and H. Kuwahara, "Compensation of pulse shape distortion due to chromatic dispersion and Kerr effect by optical phase conjugation," IEEE Photon. Technol. Lett., vol. 5, no. 10, pp. 1241-1243, Oct. 1993.

[3] G. L. Woods, P. Papaparaskeva, I. Brener, and D. A. Pitt, "Reduction of self- and cross-phase modulation-induced impairments in long-haul WDM telecommunication systems via spectral inversion," in Tech. Dig. OFC 2004, Los Angeles, CA, 2004, Paper MF66.

[4] J. Y. Huh, H. C. Ji, E. S. Son, H. S. Chung, and Y. C. Chung, "Effects of fiber parameters on performance of fiber-based OPC," in Tech. Dig. OECC 2004, Yokohama, Japan, 2004, pp. 29-30.

[5] S. Watanabe, S. Takeda, and T. Chikama, "Interband wavelength conversion of $320 \mathrm{~Gb} / \mathrm{s}(32 \times 10 \mathrm{~Gb} / \mathrm{s})$ WDM signal using a polarizationinsensitive fiber four-wave mixer," in Tech. Dig. ECOC '98, Madrid, Spain, 1998, pp. 83-87.

[6] H. C. Ji, J. H. Lee, and Y. C. Chung, "Evaluation on system outage probability due to temperature variation and statistically distributed chromatic dispersion of optical fiber," J. Lightw. Technol., vol. 22, no. 8, pp. 1893-1898, Aug. 2004.

[7] I. P. Kaminow and T. L. Koch, Optical Fiber Telecommunications IIIA. New York: Academic, 1997, ch. 8.

[8] J. Hansryd, P. A. Andrekson, M. Westlund, L. Jie, and P. O. Hedekvist, "Fiber-based optical parametric amplifiers and their applications," IEEE J. Sel. Topics Quantum Electron., vol. 8, no. 3, pp. 506-520, May/Jun. 2002.

[9] S. Wen, "Optical phase conjugation of multi-wavelength signals in a dispersion-shifted fiber," J. Lightw. Technol., vol. 15, no. 7, pp. 1061-1070, Jul. 1997. 